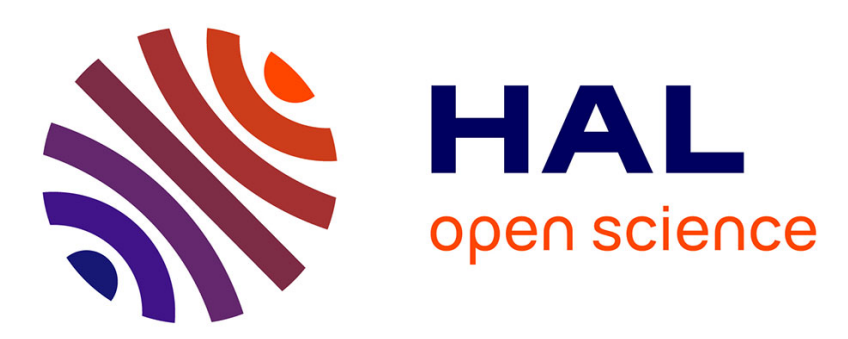

\title{
On following behaviour as a mechanism for collective movement
}

\author{
Aina Astudillo Fernandez, Jean Louis Deneubourg
}

\section{To cite this version:}

Aina Astudillo Fernandez, Jean Louis Deneubourg. On following behaviour as a mechanism for collective movement. Journal of Theoretical Biology, 2011, 284 (1), pp.7. 10.1016/j.jtbi.2011.06.001 . hal-00720865

\section{HAL Id: hal-00720865 https://hal.science/hal-00720865}

Submitted on 26 Jul 2012

HAL is a multi-disciplinary open access archive for the deposit and dissemination of scientific research documents, whether they are published or not. The documents may come from teaching and research institutions in France or abroad, or from public or private research centers.
L'archive ouverte pluridisciplinaire HAL, est destinée au dépôt et à la diffusion de documents scientifiques de niveau recherche, publiés ou non, émanant des établissements d'enseignement et de recherche français ou étrangers, des laboratoires publics ou privés. 


\section{Author's Accepted Manuscript}

On following behaviour as a mechanism for collective movement

Aina Astudillo Fernandez, Jean Louis Deneubourg

PII:

S0022-5193(11)00299-2

DOI: doi:10.1016/j.jtbi.2011.06.001

Reference: YJTBI 6502

To appear in:

Journal of Theoretical Biology

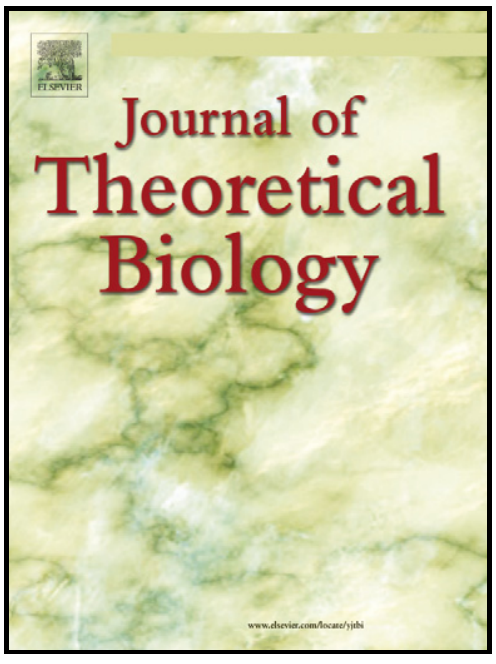

www.elsevier.com/locate/yjtbi

Received date: $\quad 7$ December 2010

Revised date: $\quad 11$ May 2011

Accepted date: 1 June 2011

Cite this article as: Aina Astudillo Fernandez and Jean Louis Deneubourg, On following behaviour as a mechanism for collective movement, Journal of Theoretical Biology, doi:10.1016/j.jtbi.2011.06.001

This is a PDF file of an unedited manuscript that has been accepted for publication. As a service to our customers we are providing this early version of the manuscript. The manuscript will undergo copyediting, typesetting, and review of the resulting galley proof before it is published in its final citable form. Please note that during the production process errors may be discovered which could affect the content, and all legal disclaimers that apply to the journal pertain. 


\title{
On following behaviour as a mechanism for collective movement
}

\author{
Aina Astudillo Fernandez ${ }^{\text {a,b }}$, Jean Louis Deneubourg ${ }^{\mathrm{a}}$ \\ ${ }^{a}$ Université libre de Bruxelles. CP 231. Bvd. du triomphe, 1050 Brussels, Belgium \\ ${ }^{b}$ Université catholique de Louvain. Pl. croix du Sud, 5, 1348, Louvain-la-Neuve, Belgium
}

Abstract

During collective movement, animals display a wide variety of mechanisms to maintain cohesion. In some species, indviduals rely mainly on following their direct predecessor, thereby forming spectacular processions of individuals in single file. Despite being the simplest case of following behaviour, it is largely absent from the theoretical literature on collective migrations. The objective of this study is to quantify the efficiency of following the predecessor, in terms of ensuring cohesion. The situation we consider is a sequence of individuals facing a bifurcation. The choice between left and right is influenced by the choice of the predecessor. First, we model this situation with a two-state Markov chain with a symmetric transition matrix. Cohesion is quantified as the expected number of individuals on either side, and the expected number of consecutive followers. Although cohesion increases with the probability of following, it remains surprisingly low unless the probability is almost equal to one. Furthermore, cohesion decreases with group size regardless of the probability of following. Then, we generalise our model to situations in which individuals have a preference for one of the two choices (asymmetric transition matrix). For some parameter sets, the tendency to

\footnotetext{
${ }^{1}$ Corresponding author: Astudillo Fernández, A.(aastudil@ulb.ac.be - Tel:+3226505119

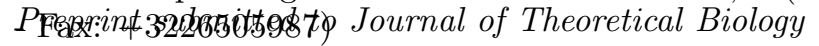


follow each other leads a large fraction of the individuals to the non preferred side. Moreover, this fraction increases with the total population size. Finally, we include the possibility to follow $N$ individuals. This provides the link between our model and other collective migration models. If enough individuals are perceived, the results shift from symmetrical (low cohesion) to asymmetrical (high cohesion) distribution of the individuals. All in all, our results suggest that following the direct predecessor must be complemented with other cohesive behaviours (involving the perception of more individuals or a navigation system) to guarantee its efficiency. We discuss our findings in the context of the different following behaviours covered in the literature. Keywords:

migration, collective decision making, group cohesion, aggregation, Markov chains

\section{Introduction}

Social decision making is common among a wide range of species. In those species, when an individual has to make a choice between a set of options, its decision is influenced by the decision of conspecifics. When imitation $^{1}$ in the broad sense is involved, it can lead to complex group behaviour (Deneubourg and Goss, 1989). This often results in some level of cohesion and/or synchronisation (Sumpter, 2006). The range of perception of each

\footnotetext{
${ }^{1}$ By imitation we mean that an individual's probability of choosing one option is increased if other individuals already chose that option. Depending on the specific context it can be referred to as allelomimesis, social facilitation, public information... but here we use it in a very broad sense.
} 
individual determines in the number of individuals by whom it will be influenced. This factor can therefore be of key importance in the resulting group cohesion.

Many types of decisions can be socially influenced. An example is temporal decisions (when to do something). For instance, pedestrians are influenced by other pedestrians as they decide when to cross the road (Faria et al., 2010). Sometimes, the immitation of others in temporal decisions can lead to synchronisation. There are countless examples ranging from flashing in fireflies (Buck, 1988, Ramırez Ávila et al., 2003) to synchronised alternation between feeding and vigilance bouts in sheep (Michelena et al., 2006, Gautrais et al., 2007) or to synchronized clapping in human audiences (Neda et al., 2000a, 2000b). Social influence has also been widely studied in the context of spatial decisions, such as where to forage, where to settle or where to breed (Danchin et al., 2004). Finally, there are directional decisions (which direction to take). Sometimes, directional decisions and temporal decisions are not dissociated. Sheep for example, tend to move when an other sheep moves, in the same direction as the initiator (Pillot et al., 2010). In this paper, however, we focus only on directional decisions, with no particular regard for the context of the displacement (where are they going to or why). More precisely, our interest lies in the mechanisms involved in the decision at the individual level, and their consequences at the collective level.

This ambition to link individual behaviour with collective movement patterns lies at the heart of a number of mathematical modelling studies. The behaviour at stake is, on the whole, following the other individuals. Understandably, the different biological realities require different types of models 
(for a review see Giardina, 2008). In flocks of birds or schools of fish, each individual repeatedly updates its position according to the position of others (Couzin and Krause, 2003). The majority of models of schooling or flocking have been individual based, self-propelled particle (SPP) models (Okubo 1986, Viscek et al., 1995, Czirok et al., 1997, Couzin et al. 2002, Grégoire and Chaté, 2004).

Another important section of the literature on collective movement concerns situations in which the directional decisions are taken sequentially by each individual, rather than simultaneously. This is typically the case of species that tend to move in single file such as social caterpillars, ants or army worms. Some fish have also been observed moving heads-to-tails (Gudger, 1944). Finally, single files occur in any species constrained to move in a sequence due to the geometry of the environment, such as sheep in mountain paths or cows in cattle-handling facilities (Grandin, 1980). The question of social influence on directional choices is typically studied with binary choice set-ups in which a bifurcation presents two identical options. The outcome of such experiments is either a binomial bell-shaped distribution (no social influence) or a U-shaped distribution, with most experiments resulting in the collective selection of one of the two options (social influence). This simple experiment has put forward collective movement in species as diverse as ants (Deneubourg et al., 1990), fish (Ward et al., 2008), dust mites (Mailleux et al., 2011), sheep (Michelena et al., 2010), caterpillars (Dussutour et al., 2008), spiders (Jeanson et al., 2004, Saffre et al., 1999) and earth worms (Zirbes et al., 2010).

The mathematical models used to describe such collective choices are 
often individual-based simulations coding for the particular mechanism involved, for example dragline attachment in the case of spiderlings (Jeanson et al., 2004). General models covering a wide range of situations have seldom been formulated. One of these general models, hereafter referred to as the trail model (Deneubourg et al.,1990) expresses the probability of choosing one option as an increasing (potentially non-linear) function of the number of individuals who previously chose that option (see section 4 for more details on this probability function). The major prediction of this model is that individuals will distribute themselves asymmetrically between the two options, if the response function is non-linear. Another, perhaps more abstract model is Polya's urn model, which makes similar predictions to the trail model (for given parameter sets). One of the assumptions of these models is that individuals are able to perceive the choice of every preceding individual. This assumption is justified in the case of many species, which use modification of the environment (e.g.. laying a physical or chemical trail) as means of communication. As long as the modification is permanent at the time-scale considered (no or little evaporation of the trail), then the assumption holds. However, with means of communication such as sight or hearing (e.g. in birds or mammals), the assumption ceases to be valid as soon as the total number of preceding individuals exceeds the range of perception.

The extreme case is when perception is limited to one individual, for example when animals in a sequence perceive others through tactile cues (contact). This is the case of processionary caterpillars (Fitzgerald, 2003), earthworms (Zirbes et al., 2010), spiny lobsters (Herrnkind, 1969, Berill 1975), or some self-assembling robots (Levi and Kernbach, 2010). In these species 
each animal follows its direct predecessor. Despite being the simplest case possible of following behaviour, it has never been studied from a theoretical point of view. Some car traffic models, called "Follow the leader" (Gazis et al., 1960), consider the influence of the direct predecessor in decisions about speed and acceleration, but not about direction.

The question we address here, is therefore how does the number of individuals perceived affect the cohesion of the moving group? We begin by developing a model for the simplest case: following a single preceding individual. We then extend the model to cover situations in which individuals follow an environmental cue as well as the preceding individual. Finally, the link between our model and the trail model is provided by a general model allowing the perception of $\mathrm{N}$ preceding individuals. Our results are discussed in terms of the efficiency of the different following behaviours covered in the literature.

\section{Following the previous individual}

The theoretical set-up is a path leading to a bifurcation. $n$ individuals have to take the path and choose between left and right, one after the other. Each individual's information is limited to its direct predecessor's choice. It has a probability $p$ of following its predecessor, and a probability $1-p$ of taking a different path from its predecessor ( $p>0.5$ by definition).

The process is memoryless, in the sense that every choice depends exclusively on the choice of the predecessor. The choices of everyone else that passed before that previous individual do not have any influence on the focal 
individual's choice. Therefore, this system can be assimilated to a two-state Markov chain, where the two states are the possible choices of the individuals (left or right). Since the probability of following the predecessor whether he took left or right is the same, the transition matrix is symmetric:

$$
\mathbf{T}=\left(\begin{array}{cc}
p & 1-p \\
1-p & p
\end{array}\right)
$$

The entry $T(i, j)$ in the transition matrix represents the probability of transition from state $i$ to state $j$. In other words, it is the probability of choosing side $j$, given that the previous individual took side $i$.

The question we address here is whether this mechanism is efficient in terms of ensuring cohesion. We suggest two ways of answering this question. Firstly, we calculate the proportion of individuals expected to end up on the side with the most individuals (hereafter referred to as the "winning side"). A very cohesive species would have an expected fraction of individuals on the winning side that is relatively high, whereas a poorly cohesive species would have an expected fraction approaching 0.5. Secondly, we calculate the number of individuals that are expected to take the same side successively, thereby forming a chain of followers. The expected length of those chains represents an accurate estimate of cohesion because all of those successive followers will end up in the same place.

Expected fraction of individuals on the winning side

Let $n$ be the total number of individuals that face the binary choice. First, we need to calculate the expected absolute number of individuals on 
the winning side. This can be done by using the definition of the expected value in probability theory $E(X)=\sum_{k=n / 2}^{n} k \cdot P(X=k)$, where $k$ can be any possible number of individuals on the winning side and ranges from $n / 2$ to $n$. $P(X=k)$ is the probability of having $k$ individuals on the winning side.

This probability is difficult to calculate, because all of the possible sequences leading to $k$ individuals on a given side do not necessarily have the same probability (see an example in Fig. 1). Therefore, we calculate this probability $P(X=k)$ by grouping all of those sequences in groups with the same number of switches (an individual taking the opposite side to his predecessor). The reason for grouping the sequences in such a way is simple: the probability of any given sequence depends only on the number of switches $a$ and on the total number of individuals $n$. Therefore all of the sequences in each group have the same probability, that is $P(X=k)=0.5 \cdot p^{a-1-s}(1-p)^{a}$. The sum of the probabilities of all those possible sequences gives us the probability of having $k$ individuals on the winning side:

$$
P(X=k)=\sum_{a=0}^{2(n-k)} 0.5 \cdot p^{n-1-a}(1-p)^{a} f(n, k, a)
$$

The function $f(n, k, a)$ gives the number of sequences leading to $k$ out of $n$ individuals on the winning side in which $a$ switches took place. We propose a way to calculate this function $f(n, k, a)$. The sequences can have two possible configurations depending on the side chosen by the first individual (Fig. 2). In the first configuration (a), the number of individuals who chose the left are divided into three subgroups, and in configuration (b) into two subgroups. For the individuals who took the right, it is the opposite. 
Fig. 3 shows the exhaustive enumeration of all the sequences in a concrete example involving 4 switches. In that example, the number of sequences of type (a) is simply the number of different ways there are to make 3 subgroups with 3 black elements multiplied by the number of ways there are to make 2 subgroups out of 4 white elements (same reasoning for type (b)).

If we define $G(x, y)$ as the number of ways to make $x$ subgroups out of $y$ elements, then the total number of sequences $f(n, k, a)$ can be calculated with the expression in Eq. 3. We give some details about this $G(x, y)$ function in appendix A. This expression was verified with simulations (appendix C).

$$
\begin{aligned}
f(n, k, a)= & 2 \overbrace{G\left(\left\lfloor\frac{a+1}{2}\right\rceil, n-k\right) \cdot G\left(\left\lfloor\frac{a+1}{2}\right\rfloor, k\right)}^{\text {sequences of type (a) }} \\
& +\overbrace{\text { sequences of type (b) }} \overbrace{\left.\left(\frac{a+1}{2}\right\rfloor, n-k\right) \cdot G\left(\left\lfloor\frac{a+1}{2}\right\rceil, k\right)}
\end{aligned}
$$

With this formula, we calculated the expected fraction of individuals on the winning side, and how it varies with the total number of individuals passing through the binary choice set-up. This was done for different values of the probability of following. As is shown in Fig. 4, the system tends towards a symmetric distribution of the individuals between the two sides, for probabilities of following that are different from 1 . In other words, even though individuals follow one another, the fraction of individuals on either side tends towards 0.5 . In Markovian terms, this is easily verified by calculating the stationary distribution of the system, that is, when the probabilities of finding the system in any given state do not change over time. At the stationary distribution the probability of finding the system in state $i$ is called $\pi(i)$ and 
is given by the formula $\pi(i)=T(j, i) /(T(j, i)+T(i, j))$, where $T(i, j)$ is the entry of the transition matrix (Eq. 1) containing the probability of taking side $j$, given that the previous individual took side $i,(i \neq j)$. In our case, $\pi($ left $)=\pi($ right $)=0.5$

\section{Expected number of consecutive followers}

The individuals that follow each other uninterruptedly form a "chain" of individuals that all end up on the same side. The mean length of those chains gives us an estimate of the cohesiveness of the species: the larger the mean chain length, the greater the cohesion. Note that this is true in general, even for choices that are not binary, which are more representative of the actual nature of most species' environment. The expected size $E(C)$ (or mean size) of these chains of followers is:

$$
E(C)=\sum_{c=1}^{n-1} c p^{c-1}(1-p)+n p^{n-1}
$$

As is shown in Fig. $5 \mathrm{~A}$, this expected chain length increases with the total number of individuals up to a certain point. From there onwards it is independent of the total number of individuals. For an infinite number of individuals, it depends exclusively on the probability of following, according to the function $E(C)=1 /(1-p)$ (Fig. 5B). It is noteworthy that the expected chain length is hardly affected by following the predecessor, except for probabilities of following superior to 0.85. For example, in a group of individuals that follow eachother with a probability of 0.85 , the expected number of followers would be of 6.7 , whereas in a group moving independently the expected number would be 2. Chains of more than 20 individuals are only 
obtained with probabilities of following superior to 0.95 .

\section{Following the previous individual and an environmental cue}

So far, we have considered the probability of following to be independent of the direction taken by the preceding individual. However, in a more general case, we can include a bias or an environmental cue that causes the individuals to prefer one side over the other. The preferred side will hereafter be referred to as the biased side. For the first individual, let the probability of taking the biased side be $b$. From then onwards, we can consider a two-state Markov process in which the Markovian transition matrix becomes asymmetric:

$$
\mathbf{T}=\left(\begin{array}{cc}
p_{B} & 1-p_{B} \\
1-p_{N B} & p_{N B}
\end{array}\right)
$$

In this transition matrix, $p_{B}$ and $p_{N B}$ are the probabilities of following the preceding individual towards the biased and the non-biased side respectively. For the system to be consistent with the scenario we propose, the parameters require some constraints: (1) the probability of following an individual towards the biased side $p_{B}$ is the highest of all probabilities (including $b$ ); (2) the probability of taking the non-biased side, even though the previous individual took the biased side $p_{N B}$ is the lowest of all probabilities (3) if the probability of following towards the non-biased side is higher than 0.5 , the attraction to others is higher than the attraction to the environmental cue causing the bias (and vice-versa). 
In this situation, the expected number of individuals on the biased side is:

$$
E(X)=\sum_{k=0}^{n} k \cdot P(K=k)
$$

The probability of having $k$ individuals on the biased side $P(K=k)$ is explained in appendix B.

We calculated the expected proportion of individuals on the biased side for different values of the three parameters $p_{B}, p_{N B}$ and $b$. Unsurprisingly, the majority of individuals always ends up on the biased side. The expected proportion tends towards a steady value as the total number of individuals increases (Fig. 6). This steady value depends on $p_{B}, p_{N B}$ (Fig. 7) and equals the probability of finding the system in state $B$, once the stationary distribution is reached, that is $\pi(B)$ :

$$
\pi(B)=\left(1-p_{N B}\right) /\left(2-p_{B}-p_{N B}\right)
$$

This value can be greater than the bias (amplification of the bias) or lower than the bias (alleviation of the bias), depending on the values of $p_{B}$ and $p_{N B}$ (Fig.6). This means that for certain parameter sets (satisfying $\pi(B)<b$ ), the tendency to follow each other can lead a large fraction of the population to the non-preferred side. At least, this fraction is larger than it would have been if the individuals were not influenced by one another. In these situations, the fraction on the non-preferred side increases with the total population. Another interesting feature is that for certain values of $p_{B}$ and $p_{N B}$, satisfying $p_{N B}=\left(2 b-b p_{B}-1\right) /(b-1)$, the expected fraction of individuals on the biased side is $b$. This result is identical to what it would 
be, had they chosen independently from one another in a biased set-up. In other words, they can be very strongly influenced by each other, and to the observer who looks only at the end result, rather than at the dynamics, it may seem as if they had just followed a bias.

\section{Following the $N$ previous individuals}

So far, we have assumed that the range of perception of indidviduals was limited to their direct predecessor. In order to allow for larger perception ranges, we generalise our model by defining $N$, the number of predecessors perceived by the focal individual. The situation we have discussed so far is in fact an extreme case where $N=1$, the other extreme being the ability to perceive all of the preceding individuals $(N=n)$, which is the case of the trail model when the trail does not evaporate.

Let us go back to a non-biased environment. Each individual perceives $N$ preceding individuals, and chooses preferentially the side where most of them went. The choice is made according to the choice function of the trail model (Deneubourg et al., 1990):

$$
p_{L}=\frac{\left(\kappa+N_{L}\right)^{\epsilon}}{\left(\kappa+N_{L}\right)^{\epsilon}+\left(\kappa+N_{R}\right)^{\epsilon}} \quad \text { and } \quad p_{R}=\frac{\left(\kappa+N_{R}\right)^{\epsilon}}{\left(\kappa+N_{L}\right)^{\epsilon}+\left(\kappa+N_{R}\right)^{\epsilon}}
$$

In this choice function, $p_{L}$ and $p_{R}$ are the probabilities of choosing the left and the right, respectively. $N_{L}$ and $N_{R}$ are the number of perceived individuals that went to the left and to the right, respectively. Parameter $\kappa$ represents the inherent attractiveness of each side. For high values of $\kappa$, the 
branches are so attractive that the attractiveness added by the social term $\left(N_{L}\right.$ or $\left.N_{R}\right)$ does not greatly influence the probability. Therefore, when $\kappa$ tends towards infinity, both probabilities tend towards 0.5 , and the resulting probability distribution is the binomial distribution. The exponent $\epsilon$ gives an estimate of the system's amplification potential. If $\epsilon=0$, the probability distribution is also binomial. This choice function was previously used to model trail following in ants (Deneubourg et al., 1990) and in dust mites (Mailleux et al. 2011). Actually, in the latter study the function used is a slightly modified version, but it shares the same properties, namely the generation of great asymmetries in the distribution of individuals when $\epsilon>1$. For this trail model, it is possible to calculate the exact probability distribution, that is the probability of all possible outcomes, expressed as the probability $p(l, r)$ of having $l$ individuals to the left and $r$ individuals to the right. The initial probabilities are $p(0,1)=p(1,0)=0.5$. The probabilities of having a negative number of individuals on either side $(p(x, y)$ with $x<0$ or $y<0)$ are naturally equal to zero. From there onwards the probability distribution is given by:

$$
p(l, r)=p(l-1, r) p_{L}+p(l, r-1) * p_{R}
$$

For numbers of perceived individuals lower than the total number of individuals, we used Monte Carlo simulations to generate the probability distributions. This was done for various values of the parameters $N, \kappa$, and $\epsilon$. Fig. 8 shows a sample of the the parameter sets we used. As expected, the lower the inherent attractiveness of the branches $\kappa$, the higher the asymmetry. Also, when $\epsilon=1$, the average number of individuals on the winning 
side is relatively low, even when many preceding individuals are perceived (high values of $N)$. Very low values of $\kappa(\kappa \leq 1)$ might make the expected number on the winning side higher, but this is due to a high probability of every individual taking the same side. In fact, for amplification to appear, the exponent $\epsilon$ has to be higher than one. As Fig. 9 shows, the asymmetries increase with the number of perceived individuals $(N)$.

\section{Discussion}

In this paper we study the efficiency (in terms of group cohesion) of following the preceding individual. This question is largely absent from the theoretical literature on collective movements and socially influenced decisions. As we show in this paper, the question is far less trivial to answer than to ask. Our main results may seem somewhat counter-intuitive: cohesive behaviour at the individual level (following the preceeding individual) does not lead to cohesion at the collective level. Eventually, it leads to a homogeneous dispersal of the group. Some other results are more intuitive, such as the positive relationship between cohesiveness and the probability of following, or the cohesion obtained from increasing the number of perceived individuals, but they remain worth quantifying. By providing a quantitative description of the relationship between parameters and variables, our model offers a set of predictions that experimenters can compare their results with. This study concerns binary-choice experiments made on groups, which are extremely popular in ethology and psychology. The set-up of these experiments (reviewed in Jeanson and Deneubourg, 2009) can either present two identical options (as in Mailleux et al. 2011, or Zirbes et al., 2010) or 
two different options (as in Canonge et al., 2009 or Ward et al. 2008). The former is an elegant way of putting forward social influence on the choice of individuals. The latter can be used to study the interplay between social interaction and individual preference. Our predictions cover both situations, and can provide insight into the interpretation of such experimental results. For example, a homogeneous distribution of individuals between two identical options is typically interpreted as an absence of social interaction. Here, we show that a homogeneous distribution can happen, even in the presence of strong social influence, provided that the perception range is limited (by the nature of the communication used, or by the experimental set-up) to a relatively small number of predecessors. Therefore, questions on perception mechanisms and population density in the set-up must be taken into account. Furthermore, the models we propose can be used to validate (or invalidate) hypotheses on the mechanisms by which animals influence one another. Take for example the hypothesis that individuals follow each other by contact. It can be tested by calculating the probability of following the direct predecessor, based on the behaviour of the second individual. From that probability and our model it is possible to predict the behaviour of the subsequent individuals (Fig. 4). The comparison between this prediction and the experimental results could confirm (or invalidate) the hypothesis. Another conclusion that can be drawn from our results is that it is useful to calculate the mean length of chains of consecutive followers. Indeed, this variable can be crucial to distinguish between a situation in which there is no social interaction and one in which each individual follows its the direct predecessor. It can also be used to make the difference between following the 
predecessor and following a trail.

The model we study in this paper is general enough to cover a wide range of situations. Animals that use tactile cues to perceive and follow their predecessor, such as processionary caterpillars (Fitzgerald, 2003), spiny lobsters (Herrnkind, 1969) or earthworms (Zirbes et al., 2010) are the obvious examples. But our predictions can extend to self-assembling robots designed to form chains, or even to humans in situations where information is limited to one's direct predecessor, such as some situations in car traffic. In fact, the model can apply to socially influenced decisions that are not directional decisions. For example, it is well known that conformism can make humans very influenced by other people's decisions (Asch, 1951). Therefore, situations in which humans have to make decisions sequentially and can perceive the decisions of others (typically consumer behaviour) can be studied through the theoretical perspective we propose.

More generally, the mathematical quetions raised in this study are relevant to any situation that can be assimilated to a two-state Markov chain. These situations can be found even outside the context of decisions. For example, a classical model to predict precipitation is a two-state Markov chain (Todorovic et al., 1975). Other studies have tackled some of the mathematical questions raised here in completely different contexts. The question of the exact expression for the probability distribution discussed in the first section (Eqs. 2 and 3) is explored from a purely mathematical point of view in the context of a correlated random walk through the integers (Renshaw and Henderson 1981). The more general case of the asymmetric environment was studied from a purely abstract perspective by Gabriel (1959). The exact 
expressions derived in these two studies are in essence very similar to ours. However, our objective here is to use these mathematical explorations to infer properties to a biological system, that is to a group of animals in motion.

One interesting feature of our results is the fragility of the cohesion of groups of followers. Indeed, we show that for probabilities of following $p<0.85$, the expected chains are not drastically longer than they would be if individuals acted independently of one another (6.7 versus 2). Furthermore, what we define as the probability of following $p$, contains in fact the probability of perceiving multiplied by the probability of following the preceding individual. In our theoretical set-up, we have implicitly considered the probability of perceiving the preceding individual to be equal to one. In natural situations, where the linear density of individuals can be low, this assumption does not necessarily hold. This potentially decreases the cohesion of groups. In addition to this, even for high probabilities of perceiving and of following (different from one), large groups will end up homogeneously distributed across the options. Cohesion actually decreases with the size of the group.

Not only does cohesion decrease with group size, but in some cases accuracy also decreases with group size. Indeed we show that in situations in which individuals have a preference for one of the options, a large fraction can end up choosing the non-preferred option, if the probability of following is large enough. More importantly, we show that this fraction increases with group size. This is directly opposed to the outcome of other types of collective decision making models, such as the trail model (Deneubourg 1990) or quorum responses (Sumpter and Pratt, 2009). In the latter, cohesiveness and 
most often accuracy increase with group size. The key difference is that in these models, there are non-linearities that arise from positive feedbacks. In our memoryless situation, it is impossible for amplification to happen, hence the importance of perceiving more than one individual, and of non-linear response functions to maintain cohesion. Indeed, as we show in section 4 , stable asymmetries in the distribution happen only when the number of perceived individuals increases and when the exponent of the probability function is higher than one.

Nevertheless, the theoretical results we present must be put into perspective with the biological reality that they represent. First of all, the cohesive species that we have talked about display behaviours that potentially increase the probability of following, or more precisely the probability of perceiving the predecessor. For example, they can synchronise departures, which increases the potential linear density. It is true that the larger the group, the lower the cohesion. Nevertheless, the groups in some cases are small enough to ensure sufficient cohesion. The smallest groupe size are groups of two, which is the case of ant recruitment tandems. This type of recruitment requires constant antennal contact between the following ant and the leading ant's abdomen (Franks and Richardson, 2006, Möglich et al 1974). It is very efficient, and most of the time both ants end up in the same food source. Caterpillars move in larger groups: Edwards (1910) reports processions of up to 300 individuals. Spiny lobsters also seem to migrate in large groups: every autumn, in the Bahamas, thousands of Spiny lobsters (Herrnkind, 1969) migrate to the South-West in parallel chains of 3 to 30 individuals (Herrnkind and Cummings, 1964). It is necessary for them to rely on complementary 
mechanisms to ensure cohesion. caterpillars use silk and chemical cues as a trail (Fitzgerald, 2003), and spiny lobsters are capable of true navigation in a magnetic field (Boles and Lohmann, 2003). However it has been shown in both cases that tactile cues are the most important cues for maintaining cohesion (Fitzgerald 2003, Herrnkind, 1969).

In this paper we formulate a model that provides answers to questions concerning the efficiency of following behaviour. By studying the simplest case possible of following behaviour, we put forward three important factors in ensuring cohesion: the number of perceived individuals, the potential for amplification, and group size.

\section{Aknowledgements}

A.A. is supported by a grant from the FRIA (Fonds pour la Formation à la Recherche dans Industrie et l'Agriculture). J.L.D. is research associate from the Belgian National Funds for Scientific Research. We also wish to thank O.Astudillo Fernandez and D.Lalor for their helpful comments and suggestions.

\section{Appendix A. The number of combinations resulting in $A$ switches}

Although intuitive, the $G(x, y)$ function requires a few definitions in order to be calculated correctly:

- There is one way to make 0 subgroups with 0 elements

- There is no way to make $x$ subgroups with $y$ elements if $x>y$

- There is no way to make 0 subgroups with $y$ elements if $y \neq 0$ 
462

463

- If $x$ and/or $y$ are negative, $G(x, y)=0$

- Otherwise, $G(x, y)=C_{x-1}^{y-1}=\frac{(y-1) !}{(y-x) !(x-1) !}$

\section{Appendix B. Generalisation to any two-state Markov chain}

Let $P_{(a)}(K=k)$ be the probability of having $k$ individuals on the biased side given that the first one chose the biased side and $P_{(b)}(K=k)$ the probability, given that the first one chose the non-biased side. The probability $P(K=k)$ of having $k$ individuals on the biased side after $n$ individuals have passed is the sum of those two probabilities.

$$
\begin{aligned}
P(K=k)= & P_{(a)}(K=k)+P_{(b)}(K=k) \\
P_{(a)}= & b \sum_{a=0}^{2(n-k)} p_{B}^{k-\left\lceil\frac{a+1}{2}\right\rceil} p_{N B}^{n-k-\left\lfloor\frac{a+1}{2}\right\rfloor}\left(1-p_{B}\right)^{\left\lceil\frac{a}{2}\right\rceil}\left(1-p_{N B}\left\lfloor^{\left\lfloor\frac{a}{2}\right\rfloor}\right.\right. \\
& G\left(\left\lfloor\frac{a+1}{2}\right\rfloor, k\right) \cdot G\left(\left\lfloor\frac{a+1}{2}\right\rfloor, n-k\right) \\
P_{(b)}= & (1-b) \sum_{a=0}^{2(n-k)} p_{B}^{k-\left\lfloor\frac{a+1}{2}\right\rfloor} p_{N B}^{n-k-\left\lceil\frac{a+1}{2}\right\rceil}\left(1-p_{B}\right)^{\left\lfloor\frac{a}{2}\right\rfloor}\left(1-p_{N B}\right)^{\left\lceil\frac{a}{2}\right\rceil} \\
& G\left(\left\lfloor\frac{a+1}{2}\right\rfloor, k\right) \cdot G\left(\left\lceil\frac{a+1}{2}\right\rceil, n-k\right)
\end{aligned}
$$

The validity of this expression was verified (appendix C).

\section{Appendix C. Verification of the exact expressions for the probabil- ity distributions}

We conducted Monte-Carlo simulations in order to verify that the exact expressions for the probability distributions in sections 1 and 2 are correct. In 
each simulation $n$ virtual individuals were sequentially faced with the choice between left and right. The probability of taking left and right depended on the choice of the preceeding individual, and on the environment (homogeneous or heterogeneous) according to the transition matrices (Eqs. 1 and 5). For each individual, a random number was generated. If the random number was lower than the probability of taking left, the individual's choice would be left, otherwise it would be right. Repeating this simulation a million times gave a good approximation of the frequency distribution of each possible outcome (analogous to a probability distribution). Fig. 10 shows that the exact expression and the simulations converge to the same distribution.

\section{References}

Asch, S. E., 1951. Effects of group pressure on the modification and distortion of judgments. In: H. Guetzkow (Ed.), Groups, leadership and men. Carnegie Press, Pittsburgh, pp.177-190

Berill, M., 1975. Gregarious behavior of juveniles of the spiny lobster, Panulirus argus (Crustacea: Decapoda). Bulletin of Marine Science 25, 515-522

Boles, L.C., Lohmann, 2003. True navigation and magnetic maps in spiny lobsters. Nature 421,60-63

Buck, J., 1988. Synchronous rhythmic flashing of fireflies.II. The Quarterly Review of Biology 63,265-289

Canonge, S., Sempo, G., Jeanson, R., Detrain, C., Deneubourg, J.L., 2009. 
Self-amplification as a source of interindividual variability: shelter selection in cockroaches. Journal of Insect Physiology 55,976-982 doi:10.1016/j.jinsphys.2009.06.011

Couzin, I.D., Krause, J., James, R., Ruxton, G.D., Franks, N.R., 2002. Collective memory and spatial sorting in animal groups. Journal of Theoretical Biology 218,1-11

Couzin, I.D., Krause, J., 2003. Self-Organization and Collective Behavior in Vertebrates. Advances in the Study of Behaviour 32,1-75 doi:10.1016/S0065$3454(03) 01001-5$

Czirok, A., Stanley, H.E., Vicsek, T., 1997. Spontaneously ordered motion of self-propelled particles. Journal of Physics A: Mathematical and General 30,1375-1385

Danchin, E., Giraldeau, L-A., Valone, T.J., Wagner, R.H., 2004. Public information: from nosy neighbours to cultural evolution. Science 305,487491

Deneubourg, J.L., Goss, S., 1989. Collective patterns and decision making. Ethology Ecology and Evolution 1,295-311

Deneubourg, J.L., Aron, S., Goss, S., Pasteels, J.M., 1990. The self-organizing exploratory pattern of the Argentine ant. Journal of Insect Behavior 3,159168

Dussutour, A., Nicolis, S.C., Despland, E., Simpson, S.J., 2008. Individual differences influence collective behaviour in social caterpillars. Animal 
517

Behaviour 76,5-16, doi:10.1016/j.anbehav.2007.12.009

Edwards, T.G., 1910. On the procession and pupation of the larva of Cnethocampa pinivora. Proceedings of the Cambridge Philosophical Society 15,431-436

Faria, J.J., Krause, S., Krause, J., 2010. Collective behavior in road crossing pedestrians: the role of social information. Behavioral Ecology 21,1236-1242

Fitzgerald, T.D., 2003. Role of trail pheromone in foraging and processionary behaviour of pine processionary caterpillars Thaumetopoea pityocampa. Journal of Chemical Ecology 29,513-532

Franks, N.R., Richardson, T., 2006. Teaching in tandem-running ants. Nature 439,153

Gabriel, K.R., 1959. The distribution of the number of successes in a sequence of dependent trials. Biometrika 46,454-460

Gautrais, J., Michelena, P., Sibbald, A., Bon, R., Deneubourg, J.L., 2007. Allelomimetic synchronization in Merino sheep. Animal Behaviour 74,14431454, doi:10.1016/j.anbehav.2007.02.020

Grandin, T., 1980. Observations of cattle behavior applied to the design of cattle-handling facilities. Applied Animal Ethology 6,19-31

Gazis, D.C., Herman, R., Rothery, R., 1960. Non-linear follow-the-leader models of traffic flow. Operations Research 9,545-567 
Giardina, I., 2008. Collective behavior in animal groups: theoretical models and empirical studies. HFSP Journal 2,205-219

Grégoire, G., Chaté, H., 2004. Onset of collective and cohesive motion. Physical Review Letters 92,025-702

Gudger, E.W., 1944. Fishes that swim heads to tails in single file. Copeia $1944,152-154$

Herrnkind, W.F., 1969. Queuing behavior of spiny lobsters. Science 164,14251427

Herrnkind, W.F, Cummings, W.C., 1964. Single file migrations of the spiny lobster Panulirus argus (Latreille). Bulletin of Marine Science of the Golf and Caribbean 14,123-125

Jeanson, R., Deneubourg, J.L., 2009. Positive feedback, convergent collective patterns and social transitions in arthropods. In: Gadau, Ü., Fewell, J. (Eds.) Organization of insect societies: From genome to sociocomplexity. Harvard University Press, pp.460-476

Jeanson, R., Deneubourg,J.L.,Theraulaz., G., 2004. Discrete dragline attachment induces aggregation in spiderlings of a solitary species. Animal Behaviour 67,531-537, doi:10.1016/j.anbehav.2003.06.013

Levi, P., Kernbach, S. (Eds.), 2010. Symbiotic multi-robot organisms. Reliability, Adaptability, Evolution Series: Cognitive Systems Monographs, 480 pp. Spinger-Verlag Berlin Heidelberg 
Mailleux, A-C., Astudillo Fernández, A., San Martin, G., Detrain, C., Deneubourg, J.L., 2011. Collective migration in house dust mites. Ethology in press

Michelena, P., Noël, S., Gautrais, J., Gerard, J-F., Deneubourg, J.L., Bon, R., 2006. Sexual dimorphism, activity budget and synchrony in groups of sheep. Oecologia 148,170-180

Michelena, P., Jeanson, R., Deneubourg, J.L., Sibbald, A., 2010. Personality and collective decision-making in foraging herbivores. Proceedings of the Royal Society B 207,1093-1099

Möglich, M., Maschwitz, U., Hölldobler, B., 1974. Tandem calling: a new kind of signal in ant communication. Science 186,1046-1047

Néda,Z., Ravasz, E.,Brechet, Y., Vicsek, T., Barabási A-L., 2000a. The sound of many hands clapping. Nature 403, 849

Néda,Z., Ravasz, E.,Vicsek, T., Brechet, Y., Barabási A-L., 2000b. Physics of the rhythmic applause. Physical Review E 61, 6987-6992

Okubo, A., 1986. Dynamical aspects of animal grouping. Advances in Biophysics 22, 1-94.

Pillot, M-H., Gautrais, J., Gouello, J., Michelena, P., Sibbald, A., Bon, R., 2010. Moving together : Incidental leaders and naïve followers. Behavioural Processes 83, 235-241 doi:10.1016/j.beproc.2009.11.006

Ramırez Ávila, M., Guisset, J.L., Deneubourg, J.L., 2003. Synchronization 
577

in light-controlled oscillators. Physica D 182,254-273

Renshaw, E., Henderson, R., 1981. The correlated random walk. Journal of Applied Probability, 18,403-414

Saffre, F. Furey, R., Krafft, B., Deneubourg, J.L., 1999. Collective decisionmaking in social spiders: dragline-mediated amplification process acts as a recruitment mechanism. Journal of Theoretical Biology 198,507-517 doi:10.1006/jtbi.1999.0927

Sumpter, D.J.T., 2006. The principles of collective animal behaviour. Philosophical Transactions of the Royal Society B 361,5-22

Sumpter, D.J.T., Pratt, S.C., 2009. Quorum responses and consensus decision making. Philosophical Transactions of the Royal Society B 364,743-753

Todorovic, P., Woolhiser, D.A., 1975. A stochastic model of n-day precipitation. Journal of applied meteorology 14,17-24

Vicsek, T., Czirok, A., Ben-Jacob, E., Cohen, I., and Shochet, O., 1995. Novel type of phase transition in a system of self-driven particles. Physical Review Letters 75,1226-1229

Ward, A.J.W., Sumpter, D.J.T., Couzin, I.D., Hart, P.J.B., Krause, J., 2008. Quorum decision-making facilitates information transfer in fish shoals. Proceedings of the National American Society 105,6948-6953

Zirbes, L., Deneubourg, J.L., Brostaux, Y., Haubruge, E., 2010. A new 
597 case of consensual decision: collective movement in earthworms. Ethology $598 \quad 116,546-55$ 
Figure 1: Sequences of 5 individuals leading to 3 individuals on the right. The sequences of individuals are represented by a sequence of squares, where each square is the choice of one individual: black means it chose the left, white means it chose the right. The different sequences can have different probabilities $(P)$, depending on the order of the squares. More precisely, the probability depends on the number of individuals that took a different path than their predecessor (number of switches $a$ ). the function $f(n, k, a)$ gives the number of sequences in each subgroup.

Figure 2: If there are four switches, it means that the first one(s) go to the left, then some go to the right, then some more to the left again ending with some on the right, or the opposite starting with the right

Figure 3: All the sequences satisfying $n=7, k=4$, and $a=4$, in which the winning side is the right. It has to be multiplied by two, in order to obtain the total number of sequences $f(n, k, a)$

Figure 4: Expected fraction of individuals on the winning side according to the total number of individuals. As the total number of individuals increases, the system tends towards symmetry (with the number on the winning side approaching 50\%)

Figure 5: Mean length of the chains according to (A.) the total number of individuals and (B.) the probability of following the preceding individual. For every probability of following different from 1, after the total number of choosers exceeds a certain size, the mean length of the chain does no longer depend on the number of choosers. This dependency is strong with probabilities higher than 0.9 . 
Figure 6: Example of the evolution of the system as individuals pass. For a given value of the bias $(b=0.6)$ and a given value of the tendency to follow the previous individual towards the biased side $\left(p_{B}=0.7\right)$, the bias can be amplified (if $\pi(B)>b$, so $p_{N B}<0.55$ ) or weakened $\left(p_{N B}>0.55\right)$

Figure 7: Proportion of individuals on the biased side for an infinite total number of passing individuals, for various values of $p_{B}$ and $p_{N B}$ and an environmental bias of $b=0.6$.

Figure 8: The expected percentage of individuals on the winning side after 2000 individuals have passed. Different sets of parameters were used in the Monte Carlo simulations. For each parameter set, we ran 2500 simulations and what appears in the figure is the average of the 2500 simulations.

Figure 9: The distribution of the simulation results for a total number of individuals $n=200$, and for a fixed attractivity of each branch $(\kappa=8)$. The distributions change as we move from one extreme $(N=1)$ to the other $(N=n=200)$. For $\epsilon=1$, the distribution remains bell shaped, but spreads around the most likely result (half of the individuals on each side). For $\epsilon=2$, however, the distribution changes from bell-shaped to U-shaped. The most likely results become the asymmetric results (most individuals on one of the two sides).

Figure 10: Probability distributions calculated with exact expressions (solid line) and with simulations (histogram). The parameters taken were $n=40, p=0.75, b=0.6, p_{B}=0.8$ and $p_{N B}=0.7$. 

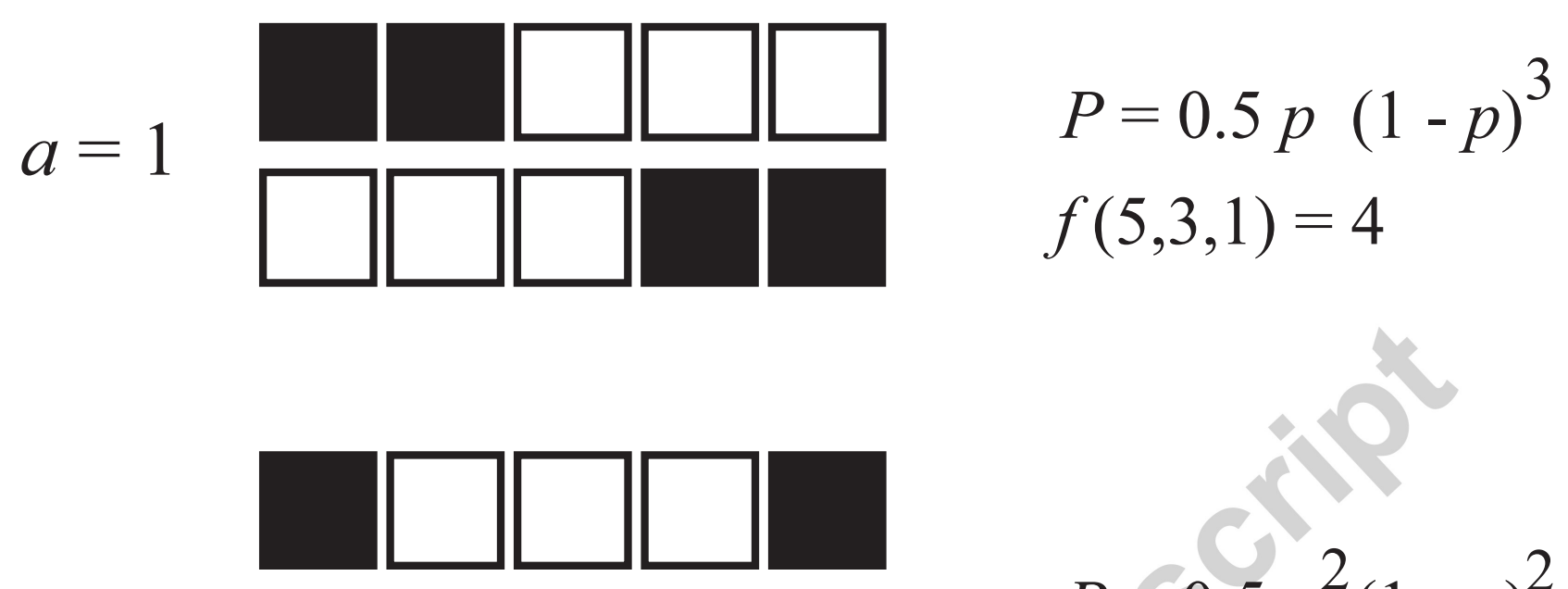

$a=2$

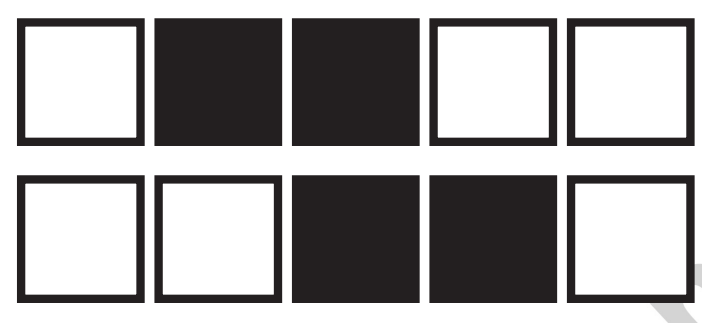

$$
\begin{aligned}
& P=0.5 p^{2}(1-p)^{2} \\
& f(5,3,2)=6
\end{aligned}
$$
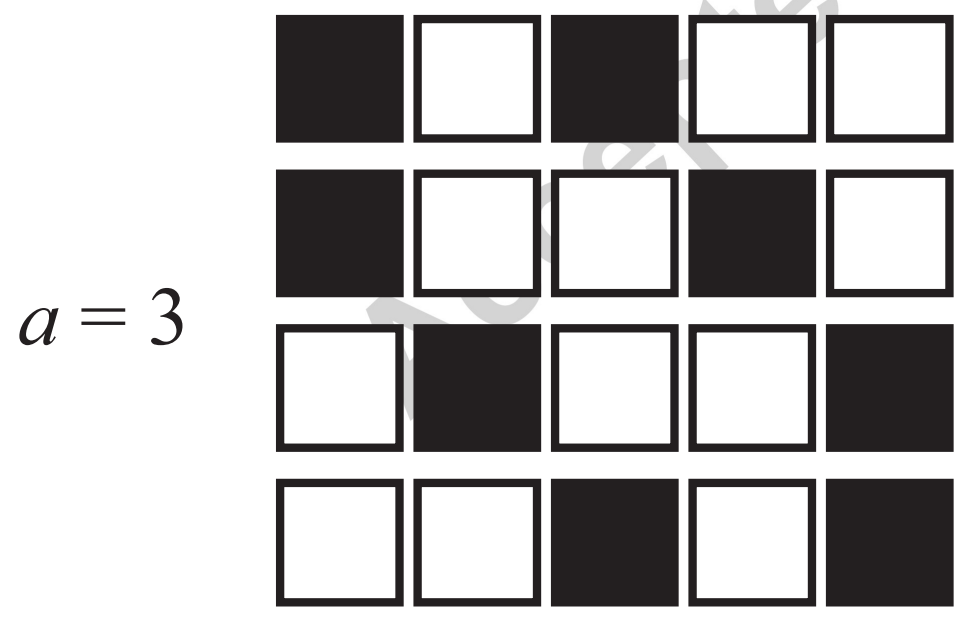

$$
\begin{aligned}
& P=0.5 p(1-p)^{3} \\
& f(5,3,3)=8
\end{aligned}
$$

$a=4$

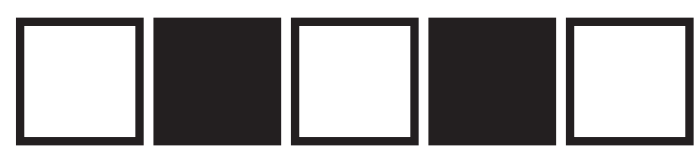

$$
\begin{aligned}
& P=0.5(1-p)^{4} \\
& f(5,3,4)=2
\end{aligned}
$$




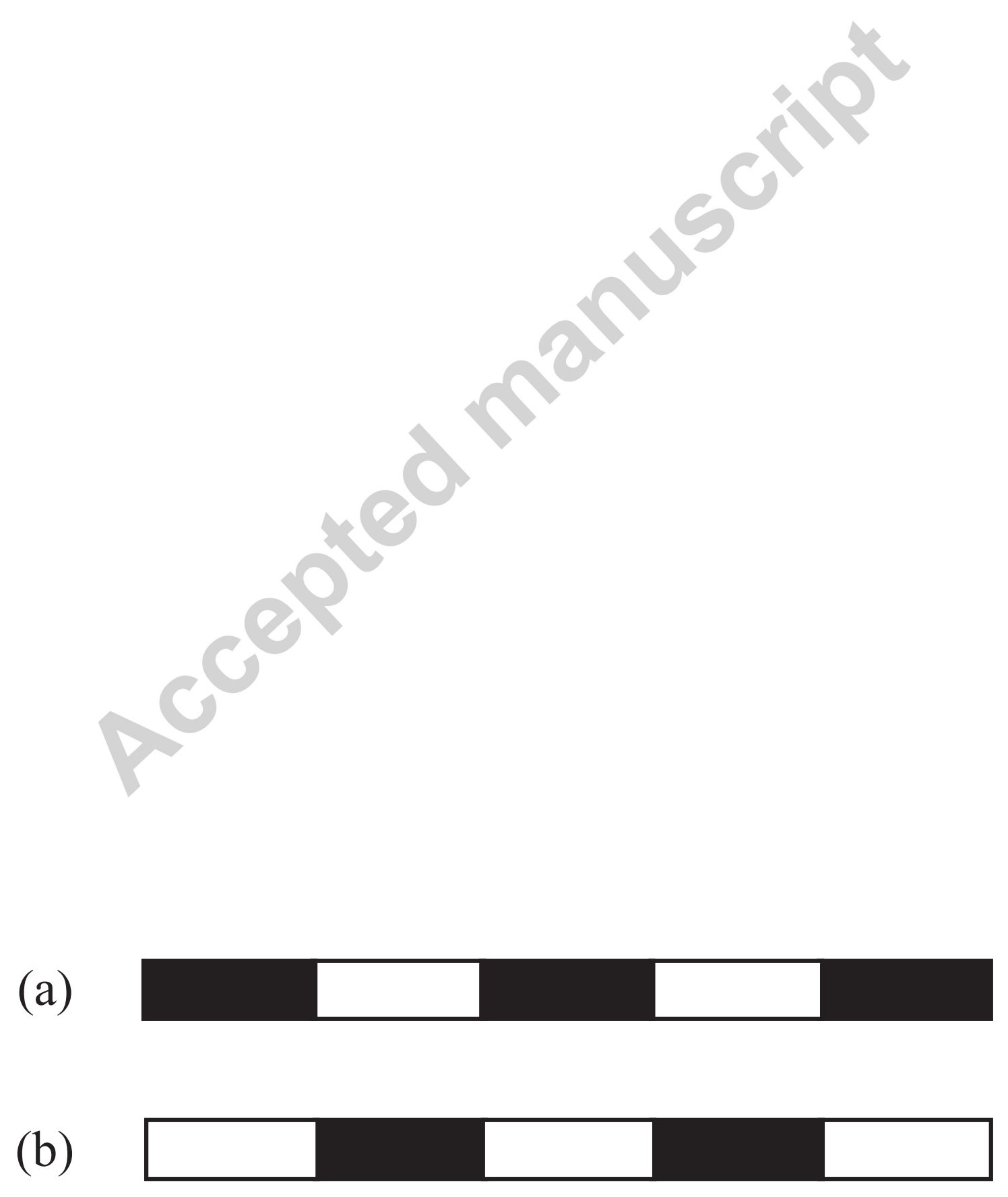

(b) 
(a)

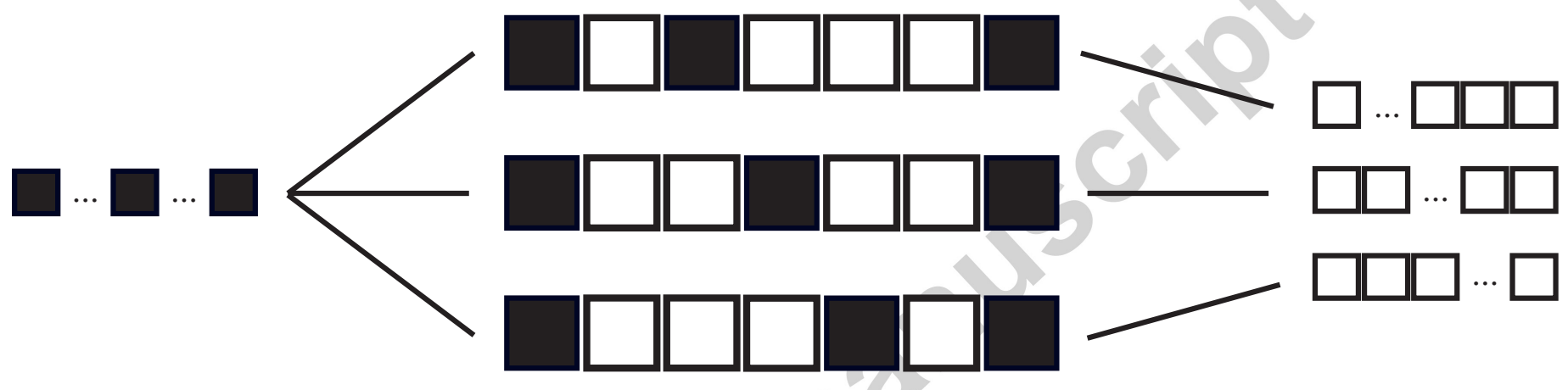

(b)

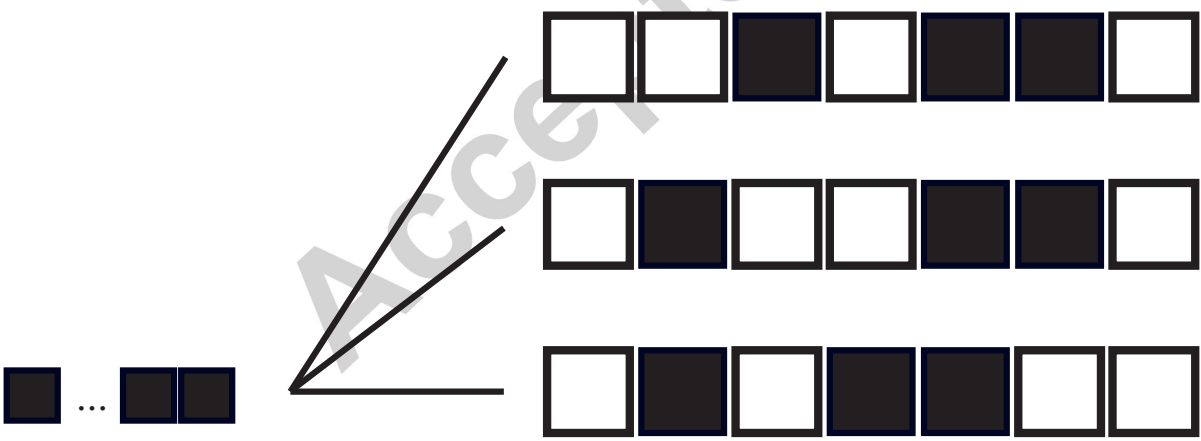

ㅁ.. $\square$
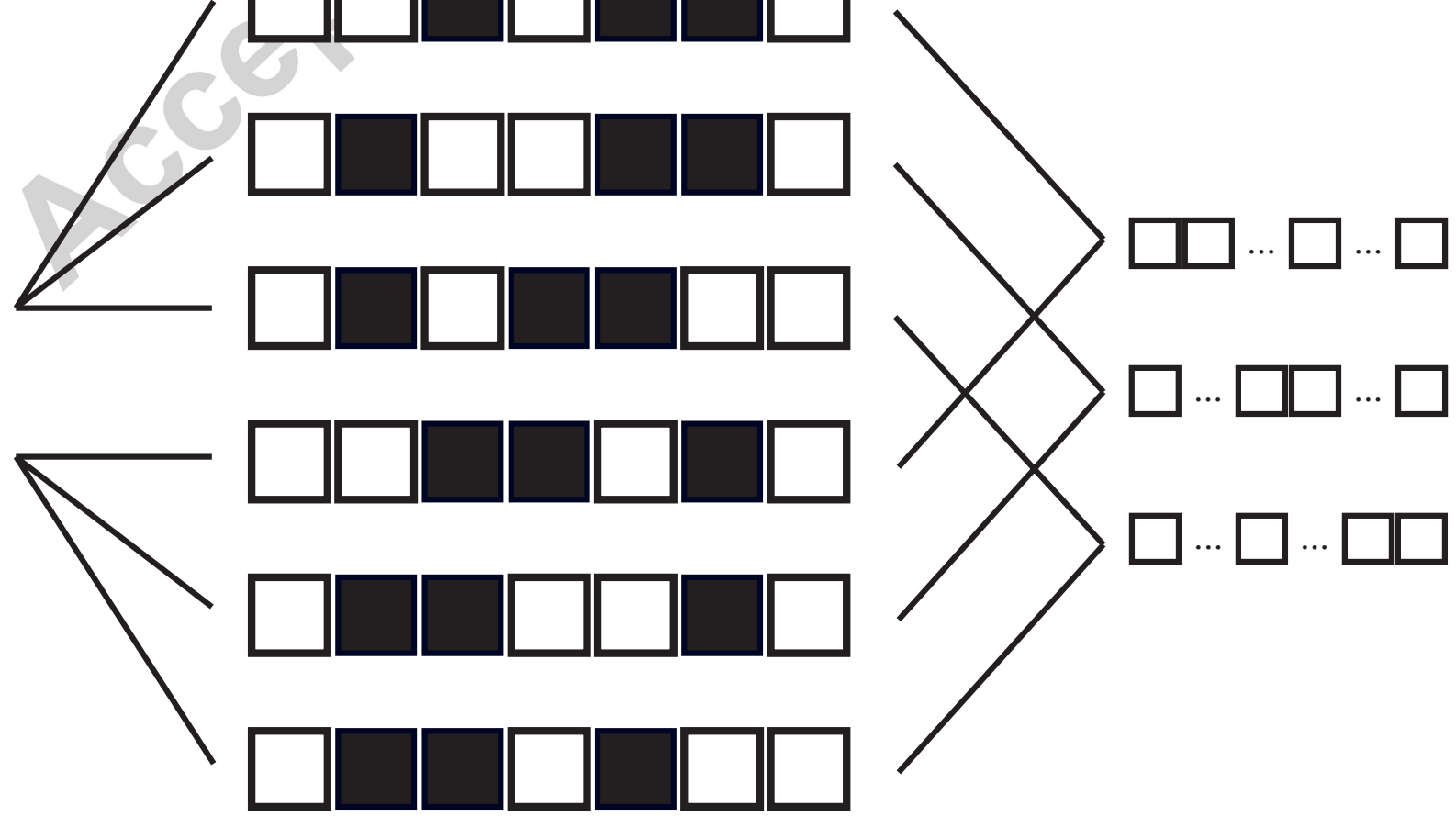


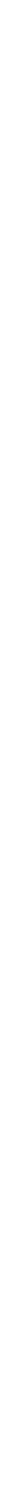

Figure 4

$p=1$

total number of individuals 
A

B
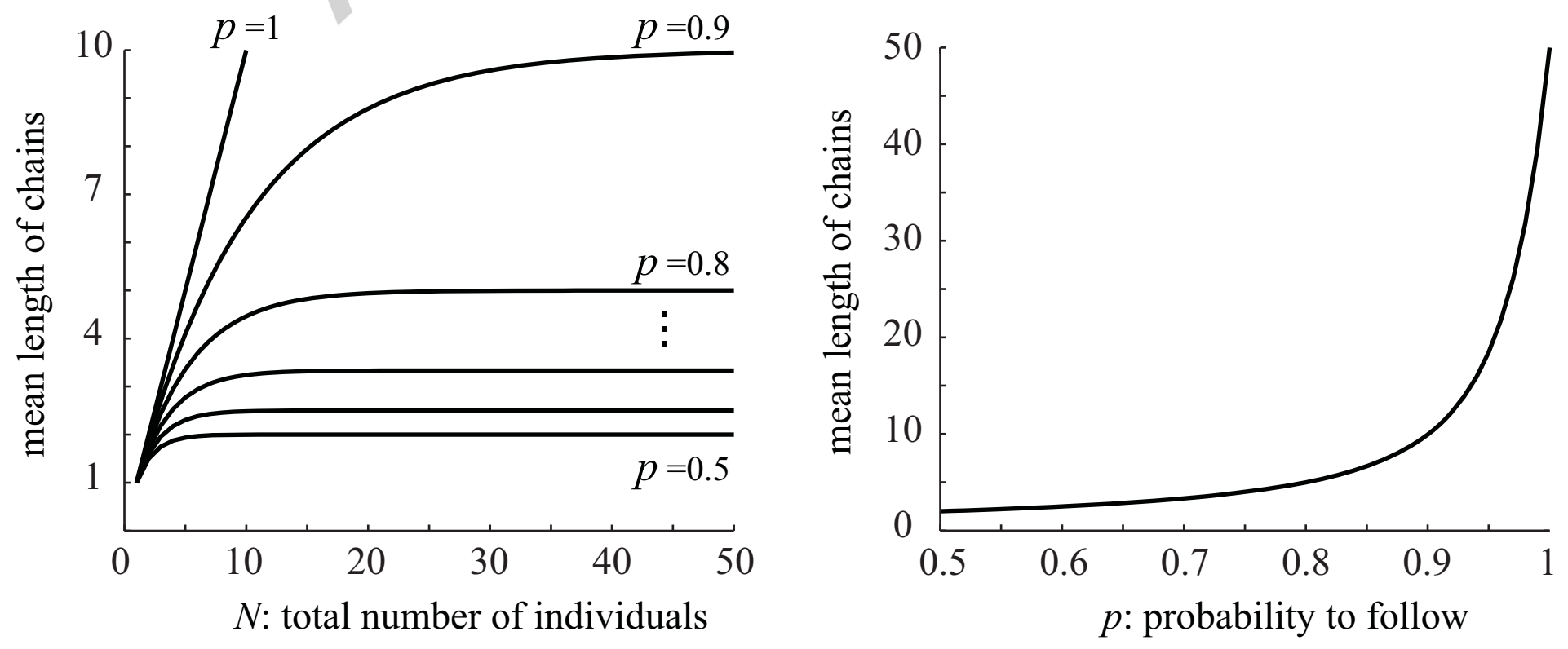


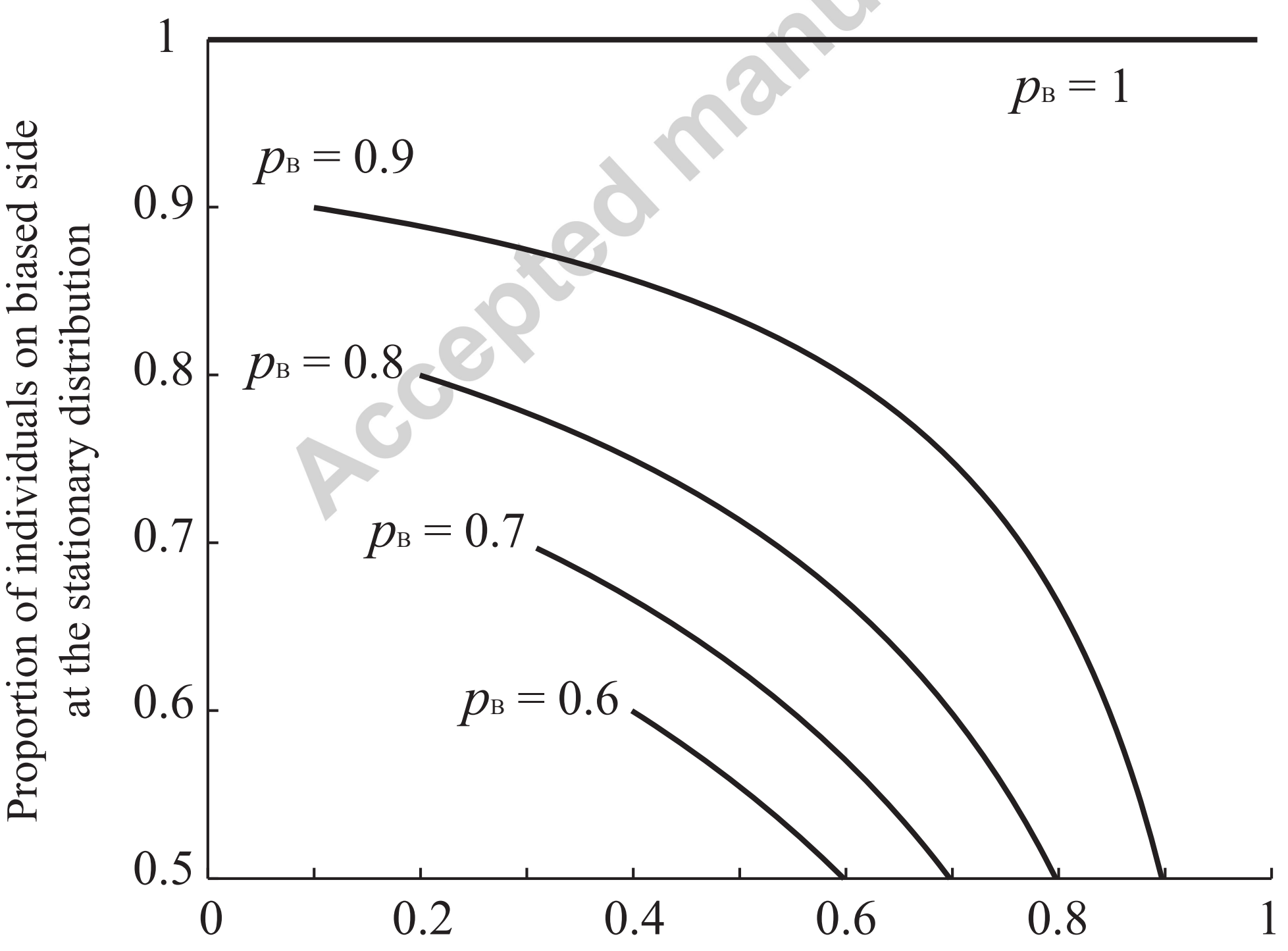

Probability of following towards the non prefered side $\left(p_{\mathrm{NB}}\right)$ 
B
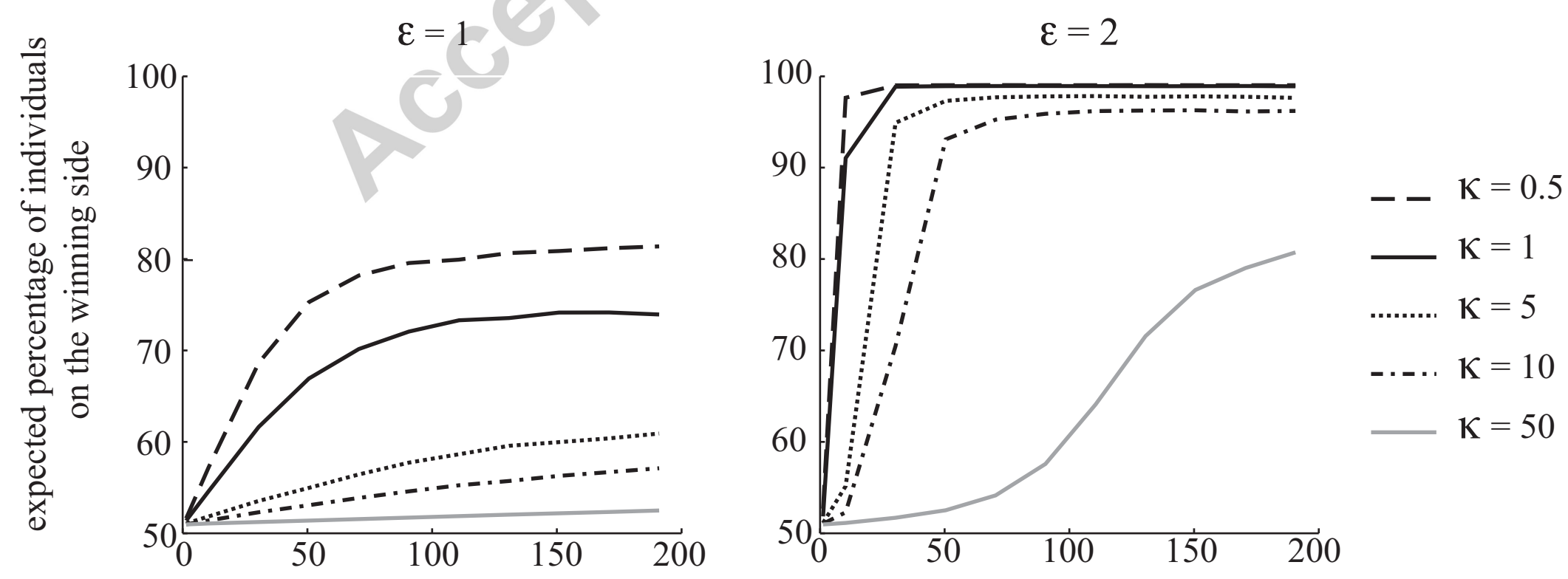

number of perceived individuals $N$ 


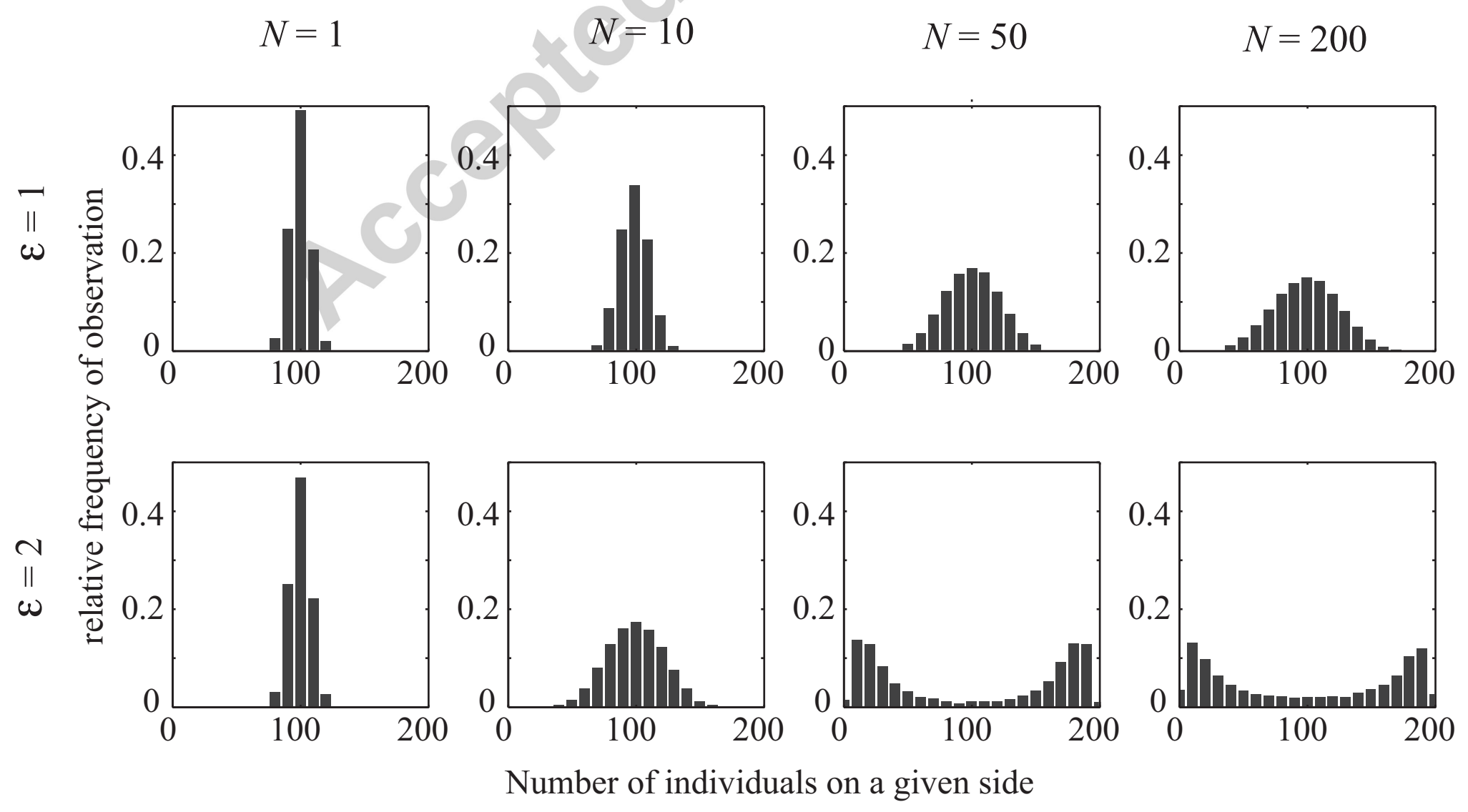




\section{Highlights}

- The potential for collective movement is evaluated in groups of indivduals that tend to follow their direct predecessor.

- This behaviour is quantified with a two-state Markov chain model.

- The results show that the cohesion resulting from following the direct predecessor is weak.

- A generalized model shows that the perception of more predecessors is needed to ensure cohesion. 